\title{
The Half-Logistic Generalized Weibull Distribution
}

\author{
Masood Anwar (D) and Amna Bibi \\ Department of Mathematics, COMSATS Institute of Information Technology, Park Road, Chak Shahzad, Islamabad, Pakistan \\ Correspondence should be addressed to Masood Anwar; masoodanwar@comsats.edu.pk
}

Received 8 August 2017; Revised 17 November 2017; Accepted 28 November 2017; Published 4 January 2018

Academic Editor: Ahmed Z. Afify

Copyright (C) 2018 Masood Anwar and Amna Bibi. This is an open access article distributed under the Creative Commons Attribution License, which permits unrestricted use, distribution, and reproduction in any medium, provided the original work is properly cited.

\begin{abstract}
A new three-parameter generalized distribution, namely, half-logistic generalized Weibull (HLGW) distribution, is proposed. The proposed distribution exhibits increasing, decreasing, bathtub-shaped, unimodal, and decreasing-increasing-decreasing hazard rates. The distribution is a compound distribution of type I half-logistic-G and Dimitrakopoulou distribution. The new model includes half-logistic Weibull distribution, half-logistic exponential distribution, and half-logistic Nadarajah-Haghighi distribution as submodels. Some distributional properties of the new model are investigated which include the density function shapes and the failure rate function, raw moments, moment generating function, order statistics, L-moments, and quantile function. The parameters involved in the model are estimated using the method of maximum likelihood estimation. The asymptotic distribution of the estimators is also investigated via Fisher's information matrix. The likelihood ratio (LR) test is used to compare the HLGW distribution with its submodels. Some applications of the proposed distribution using real data sets are included to examine the usefulness of the distribution.
\end{abstract}

\section{Introduction}

Statistical distributions are the basic aspects of all parametric statistical techniques including inference, modeling, survival analysis, and reliability. For the analysis of lifetime data, it is an important task to fit the data by a statistical model. A number of lifetime distributions have been developed in literature for this purpose. The widely used lifetime models usually have a limited range of behaviors. Such type of distributions cannot give a better fit to model all the practical situations. Recently, several authors have developed a number of families of statistical models by applying different techniques. Various techniques have been introduced in the literature to derive new flexible models as discussed by Lai [1].

Marshall and Olkin [2] introduced an effective technique to extend a family of distributions by addition of another parameter. By applying this technique, they generalized the exponential and the Weibull distributions. Al-Zahrani and Sagor [3] proposed the Poisson Lomax model by compounding the Poisson Lomax distributions. Bidram and Nadarajah [4] introduced the exponentiated EG2 distribution by using the method of resilience parameter. Kus [5] considered compounding of Poisson and exponential distribution.
Gurvich et al. [6] generalized the Weibull distribution offering a new distribution function elucidating a wide range of functional forms of the effect of size on the strength distribution, using a simple method of evaluation of the basic statistical parameters. Nadarajah and Kotz [7], Lai et al. [8], Lai et al. [9], and Xie et al. [10] further discussed some modifications of the Weibull model.

In this paper, another extension of the extended Weibull distribution is introduced using half-logistic-G generator. So we propose the half-logistic generalized Weibull (HLGW) distribution without adding any extra parameter to the baseline model. The new model is the compound distribution of two previously known distributions, one of which follows the class proposed by Gurvich et al. [6] and the other is type I half-logistic-G model. The proposed model is able to depict more complex hazard rates and provides a good alternate to the Weibull distribution that does not exhibit upside-down bathtub-shaped or unimodal failure rates.

Dimitrakopoulou et al. [11] established a three-parameter lifetime model with PDF

$$
\begin{aligned}
& g(x ; \omega, \eta, \gamma) \\
& \quad=\omega \eta \gamma x^{\eta-1}\left(1+\gamma x^{\eta}\right)^{\omega-1} \exp \left\{1-\left(1+\gamma x^{\eta}\right)^{\omega}\right\},
\end{aligned}
$$


where $x>0$ and $\omega, \eta>0$ are the shape parameters and $\gamma>0$ is a scale parameter. The CDF corresponding to (1) is

$$
G(x ; \omega, \eta, \gamma)=1-\exp \left\{1-\left(1+\gamma x^{\eta}\right)^{\omega}\right\} .
$$

In this paper, a three-parameter lifetime model is presented which is the compound model of the previously known models introduced by Dimitrakopoulou et al. [11] and half-logistic-G (HL-G) distribution called half-logistic generalized Weibull (HLGW) distribution. The half-logistic$\mathrm{G}$ distribution is presented by Cordeiro et al. [12] with the $\mathrm{CDF}$

$$
\begin{aligned}
G(x ; \gamma, \theta) & =\int_{0}^{-\ln (1-F(x ; \theta))} \frac{2 \gamma e^{-\gamma x}}{\left(1+e^{-\gamma x}\right)^{2}} d x \\
& =\frac{1-[1-F(x ; \theta)]^{\gamma}}{1+[1-F(x ; \theta)]^{\gamma}},
\end{aligned}
$$

where $F(x ; \theta)$ is the CDF of the baseline distribution and $\gamma>0$ is the shape parameter. As a special case, for $\gamma=1$, the TIHL-G is the half-logistic-G (HL-G) model with cumulative distribution function

$$
G(x ; \theta)=\frac{F(x ; \theta)}{1+\bar{F}(x ; \theta)} .
$$

The corresponding PDF to (4) is given by

$$
g(x ; \theta)=\frac{2 f(x ; \theta)}{[1+\bar{F}(x ; \theta)]^{2}},
$$

where $f(x)=(d / d x) F(x)$ and $\bar{F}(x ; \theta)=1-F(x ; \theta)$.

The rest of the paper is unfolded as follows. Section 2 contains the introduction of the half-logistic generalized Weibull (HLGW) distribution and provides the plots of its density function. Section 3 explores the distributional properties of the HLGW model. In Section 4, the method of maximum likelihood estimation is used to obtain the estimators of unknown parameters. The asymptotic distribution of the estimators is also investigated in this section via Fisher's information matrix. A simulation study is discussed in Section 5 to check out the accuracy of point and interval estimates of the HLGW parameters. Section 6 involves some applications of the HLGW distribution using lifetime data sets to examine the fitness of the proposed model. Section 7 provides concluding remarks about the paper.

\section{The Half-Logistic Generalized Weibull Distribution}

Substitution of (1) and (2) in (5) results the following PDF of the HLGW distribution:

$$
\begin{aligned}
g & (x ; \omega, \eta, \gamma) \\
& =\frac{2 \omega \eta \gamma x^{\eta-1}\left(1+\gamma x^{\eta}\right)^{\omega-1} \exp \left(1-\left(1+\gamma x^{\eta}\right)^{\omega}\right)}{\left[1+\exp \left(1-\left(1+\gamma x^{\eta}\right)^{\omega}\right)\right]^{2}},
\end{aligned}
$$

for $x>0$
The CDF associated with (6) is as follows:

$$
G(x)=\frac{1-\exp \left(1-\left(1+\gamma x^{\eta}\right)^{\omega}\right)}{1+\exp \left(1-\left(1+\gamma x^{\eta}\right)^{\omega}\right)},
$$

The parameters $\omega, \eta>0$ are the shape parameters and $\gamma>0$ is a scale parameter. From now on, a random variable $X$ with $\operatorname{PDF}(6)$ will be written as $X \sim \operatorname{HLGW}(\omega, \eta, \gamma)$.

\section{Distributional Properties}

This section deals with the investigation of the distributional properties of HLGW distribution. The statistical properties include the plots of the density function, the failure rate function, raw moments, moment generating function, order statistics, L-moments, and quantile function.

3.1. Special Cases. The HLGW distribution includes the following distributions as special cases:

(i) For $\omega=1$, the HLGW model reduces to the halflogistic Weibull (HLW) model with the PDF

$$
g(x ; \eta, \gamma)=\frac{2 \eta \gamma x^{\eta-1} \exp \left(-\gamma x^{\eta}\right)}{\left[1+\exp \left(-\gamma x^{\eta}\right)\right]^{2}},
$$

where $\eta$ is the shape parameter and $\gamma$ is the scale parameter. For $-\gamma=\alpha$, the half-logistic Weibull model is also called the power half-logistic distribution (PHLD) proposed and studied by Krishnarani [13].

(ii) For $\omega=\eta=1$, the HLGW model generates a new model, the half-logistic exponential (HLE) model, with scale parameter $\gamma$ and the PDF

$$
g(x ; \gamma)=\frac{2 \gamma \exp (-\gamma x)}{[1+\exp (-\gamma x)]^{2}} .
$$

(iii) For $\eta=1$, the HLGW model gives another new model, the half-logistic Nadarajah-Haghighi (HLNH) model, with the PDF

$$
g(x ; \omega, \gamma)=\frac{2 \omega \gamma(1+\gamma x)^{\omega-1} \exp \left(1-(1+\gamma x)^{\omega}\right)}{\left[1+\exp \left(1-(1+\gamma x)^{\omega}\right)\right]^{2}},
$$

where $\omega$ and $\gamma$ are the shape and scale parameters, respectively.

3.2. The Shapes of HLGW Distribution. The following are the conditions under which the PDF of the HLGW distribution (6) shows different behaviors:

(i) For $\eta<1$, the PDF is monotone decreasing with $\lim _{x \rightarrow 0^{+}} g(x ; \theta)=\infty, \lim _{x \rightarrow \infty} g(x ; \theta)=0$.

(ii) For $\eta=1$, the same shape is exhibited with $\lim _{x \rightarrow 0^{+}} g(x ; \theta)=\omega \gamma / 2$ and $\lim _{x \rightarrow \infty} g(x ; \theta)=0$. 


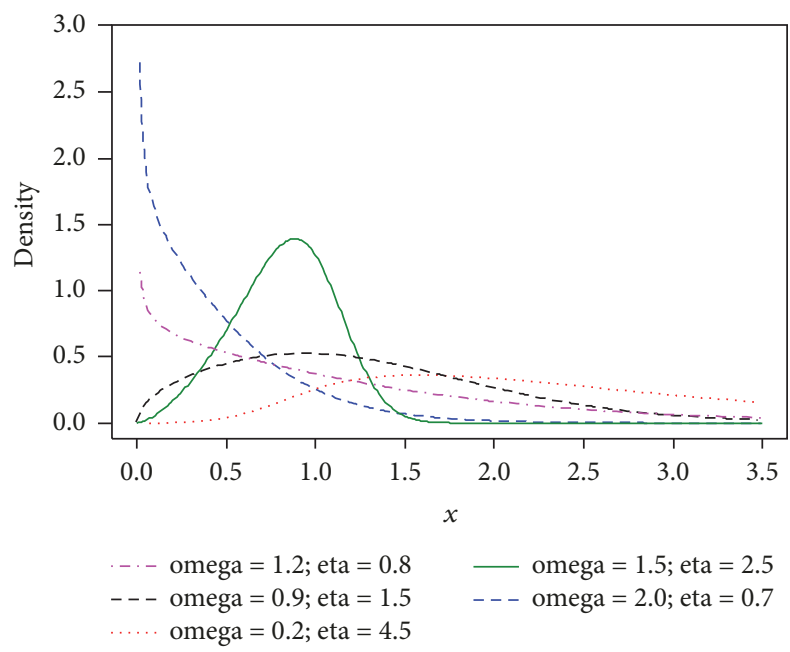

FIGURE 1: The shapes of the PDF of the HLGW distribution.

(iii) For $\eta>1$, the PDF has the value zero at the origin; then it increases to a higher value and then decreases, falling towards the value of zero at infinity.

Various behaviors of the PDF are shown in Figure 1, for some parameter values; $\gamma=1$. The mode of density (6) can be obtained from $\left.(d / d x)[\log (g(x))]\right|_{x=x_{0}}=0$.

3.3. Hazard Rate Function (HRF). For $X$ is a random variable, the HRF is given as $h(x)=g(x) / \bar{G}(x)$, where $\bar{G}=1-G$ represents the survival function given by

$$
\bar{G}(x)=\frac{2 \exp \left(1-\left(1+\gamma x^{\eta}\right)^{\omega}\right)}{1+\exp \left(1-\left(1+\gamma x^{\eta}\right)^{\omega}\right)} .
$$

The HRF of $X \sim \operatorname{HLGW}(\omega, \eta, \gamma)$ can be written as

$$
h(x ; \omega, \eta, \gamma)=\frac{\omega \eta \gamma x^{\eta-1}\left(1+\gamma x^{\eta}\right)^{\omega-1}}{\left[1+\exp \left(1-\left(1+\gamma x^{\eta}\right)^{\omega}\right)\right]},
$$

for $x>0$, demonstrating different shapes for different parameter values. By differentiating (12), it can be easily checked that

(a) for $\omega=1$ and $\eta=1$, the value of $\operatorname{HRF} h(x)$ is zero at the origin; then it increases to its maximum; after that it is constant,

(b) for $\omega \geq 1$ and $\eta>1$ or $\omega<1$ and $\eta \leq 1, h(x)$ has increasing or decreasing behavior,

(c) for $\omega \geq 1$ and $\eta<1$,

(i) if $\omega \eta \leq 1, h(x)$ is decreasing or decreasingincreasing-decreasing (DID),

(ii) if $\omega \eta>1, h(x)$ is increasing or bathtub-shaped,

(d) for $\omega \leq 1$ and $\eta>1$,

(i) if $\omega \eta<1, h(x)$ has upside-down bathtub shape (unimodal),

(ii) if $\omega \eta \geq 1, h(x)$ is monotone increasing.
The different hazard shapes are shown in Figure 2 for different parameter values. By restricting $\omega(n+1)-1 \in N$, the failure rate function (12) can also be depicted as

$$
\begin{aligned}
& h(x ; \omega, \eta, \gamma) \\
& \quad=\sum_{k=1}^{\infty} \sum_{n=0}^{\infty} \sum_{r=0}^{\omega(n+1)-1} \frac{(-1)^{n+k-1} e^{k-1}(k-1)^{n}}{(n+1) !} \gamma_{r} \eta_{r} x^{\eta_{r}-1},
\end{aligned}
$$

for $x>0$, where $\gamma_{r}=\left(\begin{array}{c}\omega(n+1) \\ r+1\end{array}\right) \gamma^{r+1}$ and $\eta_{r}=\eta(r+1)$. Thus the HRF can be written as the sum of $\omega$ terms and hence (13) is the failure rate function of a series system of $\omega$ components.

3.4. Moments. In this section, the $r$ th moment $\mu_{r}^{\prime}=E\left[X^{r}\right]$ of the HLGW model is presented as an infinite sum representation. The first four moments for $r=1,2,3,4$ have been calculated accordingly.

Theorem 1. Let $X \sim H L G W(\omega, \eta, \gamma)$ be a random variable, where $\omega, \eta, \gamma>0$, and the rth moment of $X$ about the origin is as follows:

$$
\begin{aligned}
\mu_{r}^{\prime} & =E\left(X^{r}\right) \\
& =\frac{2 \omega}{\gamma^{r / \eta}} \sum_{j=0}^{r / \eta} \sum_{k=1}^{\infty} \sum_{n=0}^{\infty}\left(\begin{array}{c}
\frac{r}{\eta} \\
j
\end{array}\right) \frac{e^{k} k^{n+1}(-1)^{j+k+n+r / \eta}}{[j+\omega(n+1)] n !}
\end{aligned}
$$

where $r=1,2,3,4$ and $r / \eta \in \mathbb{N}$.

Corollary 2. Let $X \sim H L G W(\omega, \eta, \gamma)$ be a random variable, where $\omega, \eta, \gamma>0$ and $r / \eta \in \mathbb{N}$. The first four moments of the random variable are

$$
\begin{aligned}
\mu^{\prime} & =E[X] \\
& =\frac{2 \omega}{\gamma^{1 / \eta}} \sum_{j=0}^{1 / \eta} \sum_{k=1}^{\infty} \sum_{n=0}^{\infty}\left(\begin{array}{c}
\frac{1}{\eta} \\
j
\end{array}\right) \frac{e^{k} k^{n+1}(-1)^{j+k+n+1 / \eta}}{[j+\omega(n+1)] n !} \\
\mu_{2}^{\prime} & =E\left[X^{2}\right] \\
& =\frac{2 \omega}{\gamma^{2 / \eta}} \sum_{j=0}^{2 / \eta} \sum_{k=1}^{\infty} \sum_{n=0}^{\infty}\left(\begin{array}{c}
2 \\
\eta \\
j
\end{array}\right) \frac{e^{k} k^{n+1}(-1)^{j+k+n+2 / \eta}}{[j+\omega(n+1)] n !} \\
\mu_{3}^{\prime} & =E\left[X^{3}\right] \\
& =\frac{2 \omega}{\gamma^{3 / \eta}} \sum_{j=0}^{3 / \eta} \sum_{k=1}^{\infty} \sum_{n=0}^{\infty}\left(\begin{array}{c}
\frac{3}{\eta} \\
j
\end{array}\right) \frac{e^{k} k^{n+1}(-1)^{j+k+n+3 / \eta}}{[j+\omega(n+1)] n !} \\
\mu_{4}^{\prime} & =E\left[X^{4}\right] \\
& =\frac{2 \omega}{\gamma^{4 / \eta}} \sum_{j=0}^{4 / \eta} \sum_{k=1}^{\infty} \sum_{n=0}^{\infty}\left(\begin{array}{l}
\frac{4}{\eta} \\
j
\end{array}\right) \frac{e^{k} k^{n+1}(-1)^{j+k+n+4 / \eta}}{[j+\omega(n+1)] n !} .
\end{aligned}
$$

3.5. Moment Generating Function (MGF). The MGF of $X$ is retrieved from $M_{X}(t)=E\left[e^{t X}\right]=\int e^{t x} g(x) d x$. 

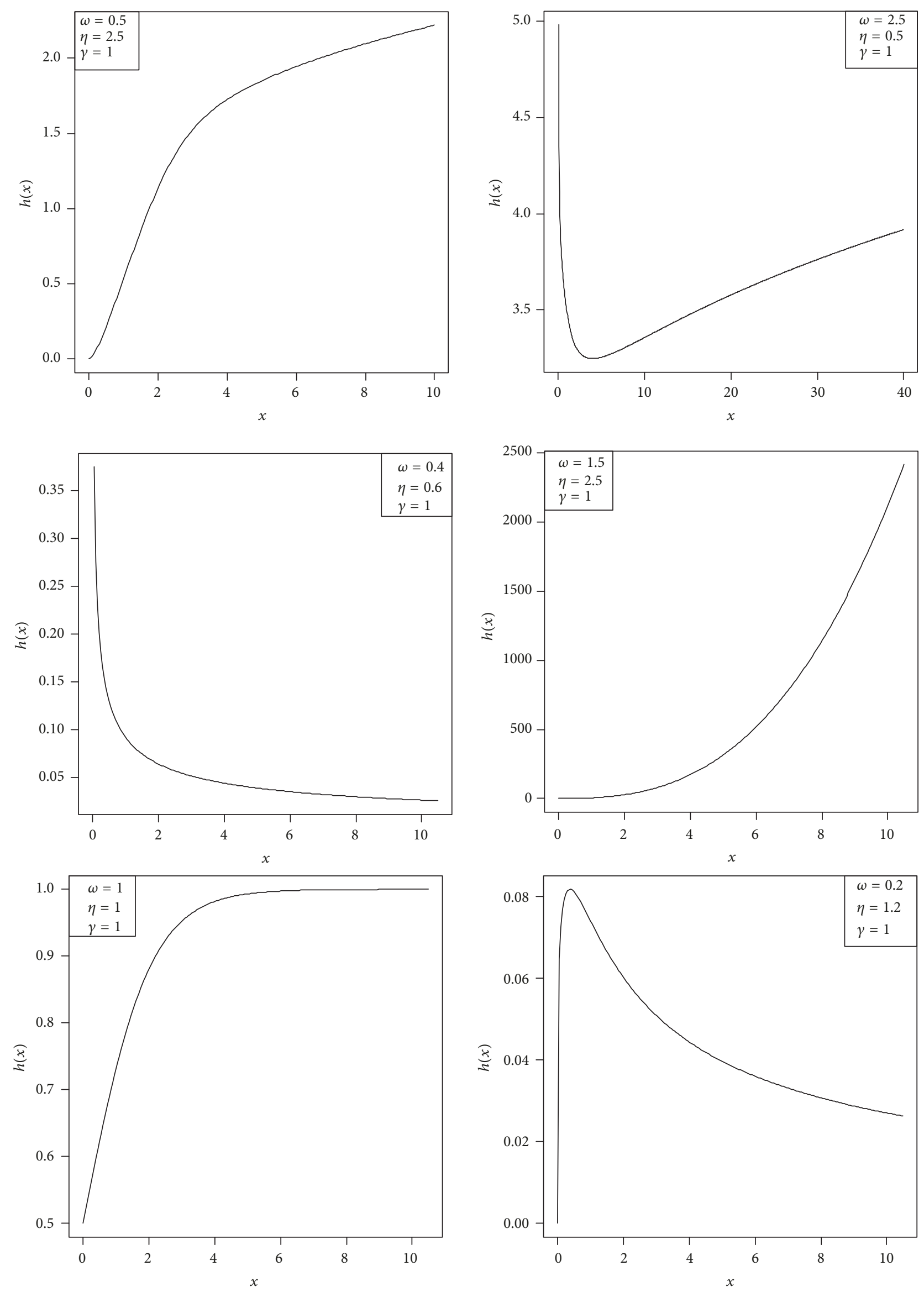

Figure 2: Continued. 


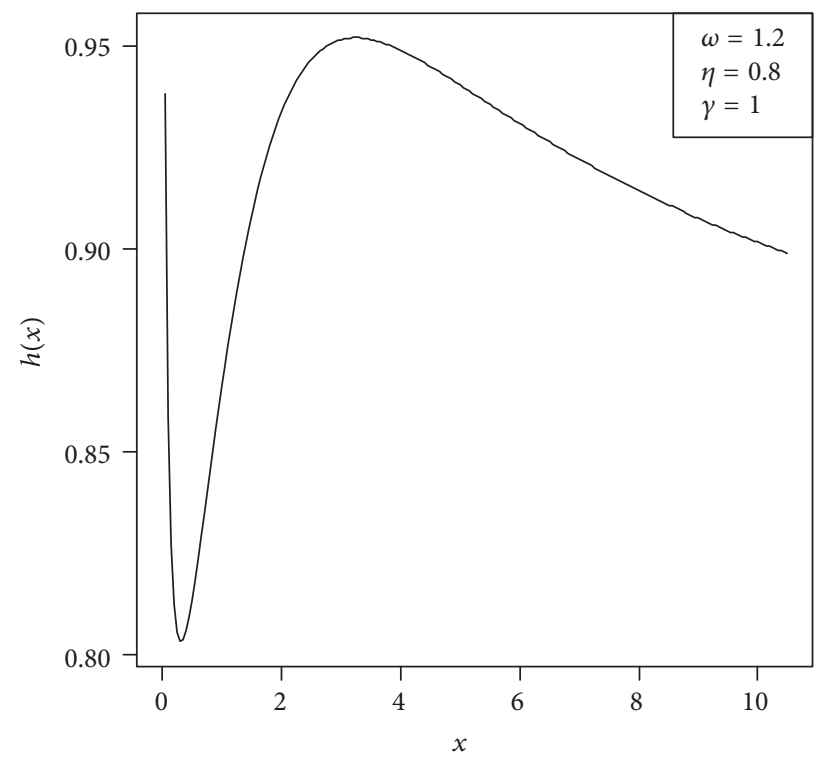

FIGURE 2: The HRF of HLGW distribution for different parameter values.

Theorem 3. let $X \sim H L G W(\omega, \eta, \gamma)$ be a random variable, and the moment generating function (MGF) of $X$ is given by

$$
\begin{aligned}
& M_{X}(t)=\frac{2 \omega}{\gamma^{j / \eta}} \\
& \quad \cdot \sum_{k=1}^{\infty} \sum_{n=0}^{\infty} \sum_{j=0}^{n} \sum_{i=0}^{j / \eta}\left(\begin{array}{l}
n \\
j
\end{array}\right)\left(\begin{array}{c}
\frac{j}{\eta} \\
i
\end{array}\right) \frac{e^{k} k^{n-j+1} t^{j}(-1)^{i+j+k+n+j / \eta}}{[i+\omega(n-j+1)] n !}
\end{aligned}
$$

where $j / \eta \in \mathbb{N}$.

3.6. Order Statistics. Let $X_{1}, X_{2}, \ldots, X_{m}$ be a random sample of size $m$ from a distribution with PDF $g(x)$ and CDF $G(x)$ and $X_{1: m}, X_{2: m}, \ldots, X_{m: m}$ are the analogous order statistics. The PDF and CDF of $X_{r: m}, 1 \leqslant r \leqslant m$, are

$$
\begin{gathered}
g_{r: m}(x)=\frac{1}{B(r, m-r+1)} g(x)[G(x)]^{r-1} \\
\cdot[1-G(x)]^{m-r}=\frac{1}{B(r, m-r+1)} g(x) \\
\cdot \sum_{i=0}^{m-r}\left(\begin{array}{c}
m \\
j
\end{array}\right)\left(\begin{array}{c}
m-r \\
i
\end{array}\right)(-1)^{i}[G(x)]^{i+r-1}, \\
G_{r: m}=\sum_{k=r}^{m}\left(\begin{array}{c}
m \\
k
\end{array}\right)[G(x)]^{k}[1-G(x)]^{m-k},
\end{gathered}
$$

where $B(r, m-r+1)$ is the beta function.

Theorem 4. Let $g(x)$ and $G(x)$ be the PDF and CDF of random variable $X \sim H L G W(\omega, \eta, \gamma)$; then the PDF of $X_{r: m}$ is

$$
g_{r: m}(x)=2 \omega \eta \gamma C_{r: m} \sum_{i=0}^{m-r}\left(\begin{array}{c}
m-r \\
i
\end{array}\right)(-1)^{i}
$$

$$
\begin{aligned}
& \frac{x^{\eta-1}\left(1+\gamma x^{\eta}\right)^{\omega-1} \exp \left[1-\left(1+\gamma x^{\eta}\right)^{\omega}\right]}{\left[1+\exp \left(1-\left(1+\gamma x^{\eta}\right)^{\omega}\right)\right]^{2}} \\
& \times\left[\frac{1-\exp \left(1-\left(1+\gamma x^{\eta}\right)^{\omega}\right)}{1+\exp \left(1-\left(1+\gamma x^{\eta}\right)^{\omega}\right)}\right]^{i+r-1},
\end{aligned}
$$

where $C_{r: m}=B[(r, m-r+1)]^{-1}$.

The CDF corresponding to (18) is

$$
\begin{aligned}
G_{r: m}(x)= & \sum_{k=r}^{m}\left(\begin{array}{c}
m \\
k
\end{array}\right)\left[\frac{1-\exp \left(1-\left(1+\gamma x^{\eta}\right)^{\omega}\right)}{1+\exp \left(1-\left(1+\gamma x^{\eta}\right)^{\omega}\right)}\right]^{k} \\
& \cdot\left[\frac{2 \exp \left(1-\left(1+\gamma x^{\eta}\right)^{\omega}\right)}{1+\exp \left(1-\left(1+\gamma x^{\eta}\right)^{\omega}\right)}\right]^{m-k}
\end{aligned}
$$

3.7. L-Moments. Suppose that we have a random sample $X_{1}, X_{2}, \ldots, X_{n}$ collected from $X \sim \operatorname{HLGW}(\omega, \eta, \gamma)$. The $r$ th population L-moments are as follows:

$$
\begin{aligned}
E\left[X_{r: n}\right]=\int_{0}^{\infty} x g\left(X_{r: n}\right) d x=\int_{0}^{\infty} x \sum_{i=0}^{n-r}\left(\begin{array}{c}
n-r \\
i
\end{array}\right)(-1)^{i} \\
\quad \cdot \frac{2 \omega \eta \gamma C_{r: n} x^{\eta-1}\left(1+\gamma x^{\eta}\right)^{\omega-1} e^{1-\left(1+\gamma x^{\eta}\right)^{\omega}}}{\left(1+e^{1-\left(1+\gamma x^{\eta}\right)^{\omega}}\right)^{2}} \\
\quad \times\left(\frac{1-e^{1-\left(1+\gamma x^{\eta}\right)^{\omega}}}{1+e^{1-\left(1+\gamma x^{\eta}\right)^{\omega}}}\right)^{i+r-1} d x .
\end{aligned}
$$

We use the substitution $y=1+\gamma x^{\eta}$, so $x=((y-1) / \gamma)^{1 / \eta}$ and $d x=(1 / \gamma \eta)((y-1) / \gamma)^{1 / \eta-1} d y$, where $1 / \eta \in \mathbb{N}$. Therefore, we get 


$$
\begin{gathered}
E\left[X_{r: n}\right]=2 \omega C_{r: n} \sum_{i=0}^{n-r} \sum_{k=0}^{i+r-1} \sum_{j=0}^{\infty} \sum_{l=0}^{1 / \eta} \sum_{m=0}^{\infty}\left(\begin{array}{c}
n-r \\
i
\end{array}\right)\left(\begin{array}{c}
i+r-1 \\
k
\end{array}\right)\left(\begin{array}{c}
-(i+r+1) \\
j
\end{array}\right)\left(\begin{array}{c}
\frac{1}{\eta} \\
l
\end{array}\right) \times\left(\frac{-1}{\gamma}\right)^{1 / \eta} \\
\cdot \frac{(-1)^{j+k+l+m} e^{j+k+1}(j+k+1)^{m}}{m !} \int_{1}^{\infty} y^{l+m \omega+\omega-1} d y .
\end{gathered}
$$

By working out the integration, we arrive at the following formula:

$$
\begin{aligned}
& E\left[X_{r: n}\right] \\
& =2 \omega \sum_{i=0}^{n-r} \sum_{k=0}^{i+r-1} \sum_{j=0}^{\infty} \sum_{l=0}^{1 / \eta} \sum_{m=0}^{\infty}\left(\frac{-1}{\gamma}\right)^{1 / \eta} \frac{(j+k+1)^{m} e^{j+k+1}}{l+\omega(m+1) m !} A_{i j k l},
\end{aligned}
$$

where

$$
\begin{aligned}
& A_{i j k l}=C_{r: n}(-1)^{j+k+l+m+1} \\
& \quad \cdot\left(\begin{array}{c}
n-r \\
i
\end{array}\right)\left(\begin{array}{c}
i+r-1 \\
k
\end{array}\right)\left(\begin{array}{c}
-(i+r+1) \\
j
\end{array}\right)\left(\begin{array}{l}
\frac{1}{\eta} \\
l
\end{array}\right) .
\end{aligned}
$$

The relation (22) can be used to find out the first L-moments of $X_{r: n}$; that is, for $r=n=1$ we get $\lambda_{1}=E\left[X_{1: 1}\right]$.

$$
\begin{gathered}
\lambda_{1}=2 \omega \sum_{j=0}^{\infty} \sum_{m=0}^{\infty} \sum_{l=0}^{1 / \eta}\left(\begin{array}{c}
-2 \\
j
\end{array}\right)\left(\begin{array}{c}
\frac{1}{\eta} \\
l
\end{array}\right)\left(\frac{-1}{\gamma}\right)^{1 / \eta} \\
\cdot \frac{(-1)^{j+l+m+1} e^{j+1}(j+1)^{m}}{(l+\omega(m+1)) m !} .
\end{gathered}
$$

The other two moments, $\lambda_{2}$ and $\lambda_{3}$, are, respectively, given by

$$
\begin{aligned}
\lambda_{2} & =4 \omega \sum_{j=0}^{\infty} \sum_{l=0}^{1 / \eta} \sum_{m=0}^{\infty}\left(\begin{array}{l}
\frac{1}{\eta} \\
l
\end{array}\right) \frac{(-1 / \gamma)^{1 / \eta}}{(l+\omega(m+1)) m !} \\
& \times\left[\sum_{i=0}^{1} \sum_{k=0}^{i}\left(\begin{array}{l}
1 \\
i
\end{array}\right)\left(\begin{array}{l}
i \\
k
\end{array}\right)\left(\begin{array}{c}
-2-\mathrm{i} \\
j
\end{array}\right)(-1)^{j+k+l+m+1}\right. \\
& \cdot e^{j+k+l}(j+k+l)^{m}+\sum_{k=0}^{1}\left(\begin{array}{l}
1 \\
k
\end{array}\right)\left(\begin{array}{c}
-3 \\
j
\end{array}\right)(-1)^{j+k+l+m+1} \\
& \left.\cdot e^{j+k+1}(j+k+1)^{m}\right] \cdot \\
\lambda_{3}= & 2 \omega \sum_{j=0}^{\infty} \sum_{l=0}^{1 / \eta} \sum_{m=0}^{\infty}\left(\begin{array}{c}
1 \\
\eta \\
l
\end{array}\right) \\
& \cdot \frac{(-1 / \gamma)^{1 / \eta}(-1)^{j+k+l+m+1} e^{j+k+1}(j+k+1)^{m}}{(l+\omega(m+1)) m !}
\end{aligned}
$$

$$
\begin{aligned}
& \times\left[\sum_{i=0}^{2} \sum_{k=0}^{i}\left(\begin{array}{l}
2 \\
i
\end{array}\right)\left(\begin{array}{l}
i \\
k
\end{array}\right)\left(\begin{array}{c}
-2-i \\
j
\end{array}\right)\right. \\
& +3 ! \sum_{i=0}^{1} \sum_{k=0}^{i+1}\left(\begin{array}{l}
1 \\
i
\end{array}\right)\left(\begin{array}{c}
i+1 \\
k
\end{array}\right)\left(\begin{array}{c}
-3-i \\
j
\end{array}\right) \\
& \left.+3 \sum_{k=0}^{2}\left(\begin{array}{l}
2 \\
k
\end{array}\right)\left(\begin{array}{c}
-4-i \\
j
\end{array}\right)\right]
\end{aligned}
$$

3.8. Quantile Function. For $X$ to be a random variable with the PDF (6), the quantile function $Q(u)$ is

$$
Q(u)=\inf \{x \in R: G(x) \geqslant u\}, \quad \text { where } 0<u<1 \text {. }
$$

The above relation is used to find the quantile function of HLGW distribution. Therefore, we have

$$
Q(u)=\left[\frac{1}{\gamma}\left\{\left(1-\ln \left(\frac{1-u}{1+u}\right)\right)^{1 / \omega}-1\right\}\right]^{1 / \eta}
$$

Hence, the generator for $X$ can be given by the following algorithm:

(1) Generate $U \sim$ uniform $(0,1)$.

(2) Use (27) and obtain an outcome of $X$ by $X=Q(U)$.

By using the quantile function (27), we can examine the Bowley skewness [14] and Moors kurtosis [15] for HLGW as follows:

$$
\begin{aligned}
& s k=\frac{Q(3 / 4)+Q(1 / 4)-2 Q(2 / 4)}{Q(3 / 4)-Q(1 / 4)} \\
& \kappa=\frac{Q(3 / 8)-Q(1 / 8)+Q(7 / 8)-Q(5 / 8)}{Q(3 / 4)-Q(1 / 4)} .
\end{aligned}
$$

Table 1 illustrates the values of skewness and kurtosis for the HLGW model for some values of $\omega, \eta$, and $\gamma$. It can be noted that the skewness and kurtosis are free of parameter $\gamma$ and they are decreasing functions of the parameters $\omega$ and $\eta$. 
TABLE 1: Skewness and kurtosis of HLGW for different values of $\omega, \eta$, and $\gamma$.

\begin{tabular}{|c|c|c|c|c|c|c|c|}
\hline \multirow{2}{*}{$\gamma$} & \multirow{2}{*}{$\omega$} & \multicolumn{2}{|c|}{$\eta=0.5$} & \multicolumn{2}{|c|}{$\eta=1.0$} & \multicolumn{2}{|c|}{$\eta=2.0$} \\
\hline & & sk & $k u$ & sk & $k u$ & sk & $k u$ \\
\hline \multirow{5}{*}{0.5} & 0.5 & 0.6530 & 2.4642 & 0.3366 & 1.4540 & 0.1303 & 1.2492 \\
\hline & 1.0 & 0.4634 & 1.6286 & 0.1808 & 1.2395 & 0.0197 & 1.2074 \\
\hline & 1.5 & 0.3913 & 1.4463 & 0.1264 & 1.1952 & -0.0196 & 1.2045 \\
\hline & 2.0 & 0.3541 & 1.3700 & 0.0989 & 1.1779 & -0.0396 & 1.2054 \\
\hline & 2.5 & 0.3314 & 1.3286 & 0.0824 & 1.1691 & -0.0517 & 1.2067 \\
\hline \multirow{5}{*}{1.5} & 0.5 & 0.6530 & 2.4642 & 0.3366 & 1.4540 & 0.1303 & 1.2492 \\
\hline & 1.0 & 0.4634 & 1.6286 & 0.1808 & 1.2395 & 0.0197 & 1.2074 \\
\hline & 1.5 & 0.3913 & 1.4463 & 0.1264 & 1.1952 & -0.0196 & 1.2045 \\
\hline & 2.0 & 0.3541 & 1.3700 & 0.0989 & 1.1779 & -0.0396 & 1.2054 \\
\hline & 2.5 & 0.3314 & 1.3286 & 0.0824 & 1.1691 & -0.0517 & 1.2067 \\
\hline \multirow{5}{*}{2.0} & 0.5 & 0.6530 & 2.4642 & 0.3366 & 1.4540 & 0.1303 & 1.2492 \\
\hline & 1.0 & 0.4634 & 1.6286 & 0.1808 & 1.2395 & 0.0197 & 1.2074 \\
\hline & 1.5 & 0.3913 & 1.446 & 0.1264 & 1.1952 & -0.0196 & 1.2045 \\
\hline & 2.0 & 0.3541 & 1.3700 & 0.0989 & 1.1779 & -0.0396 & 1.2054 \\
\hline & 2.5 & 0.3314 & 1.3286 & 0.0824 & 1.1691 & -0.0517 & 1.2067 \\
\hline \multirow{5}{*}{3.0} & 0.5 & 0.6530 & 2.4642 & 0.3366 & 1.4540 & 0.1303 & 1.2492 \\
\hline & 1.0 & 0.4634 & 1.6286 & 0.1808 & 1.2395 & 0.0197 & 1.2074 \\
\hline & 1.5 & 0.3913 & 1.4463 & 0.1264 & 1.1956 & -0.0196 & 1.2045 \\
\hline & 2.0 & 0.3541 & 1.3700 & 0.0989 & 1.1779 & -0.0396 & 1.2054 \\
\hline & 2.5 & 0.3314 & 1.3286 & 0.0824 & 1.1691 & -0.0517 & 1.2067 \\
\hline
\end{tabular}

\section{Estimation}

The log-likelihood function is expressed as

$$
\begin{aligned}
\ell(x ; \omega, \eta, \gamma) \\
=n \ln (2)+n \ln (\omega)+n \ln (\eta)+n \ln (\gamma) \\
\quad+(\eta-1) \sum_{i=1}^{n} \ln x_{i}+(\omega-1) \sum_{i=1}^{n} \ln \left(1+\gamma x_{i}^{\eta}\right) \\
\quad+\sum_{i=1}^{n}\left(1-\left(1+\gamma x_{i}^{\eta}\right)^{\omega}\right) \\
\quad-2 \sum_{i=1}^{n} \ln \left[1+\exp \left(1-\left(1+\gamma x_{i}^{\eta}\right)^{\omega}\right)\right] .
\end{aligned}
$$

Taking the first partial derivatives of $\ell(x ; \omega, \eta, \gamma)$ with respect to $\omega, \eta$, and $\gamma$ and letting them equal zero, we obtain a nonlinear system of equations.

$$
\begin{aligned}
\frac{\partial \ell}{\partial \omega}= & \frac{n}{\omega}+\sum_{i=1}^{n} \ln \left(1+\gamma x_{i}^{\eta}\right) \\
& -\sum_{i=1}^{n}\left(1+\gamma x_{i}^{\eta}\right)^{\omega} \ln \left(1+\gamma x_{i}^{\eta}\right) \\
& +2 \sum_{i=1}^{n} \frac{\left(1+\gamma x_{i}^{\eta}\right)^{\omega} \ln \left(1+\gamma x_{i}^{\eta}\right) e^{1-\left(1+\gamma x_{i}^{\eta}\right)^{\omega}}}{1+e^{1-\left(1+\gamma x_{i}^{\eta}\right)^{\omega}}}=0 . \\
\frac{\partial \ell}{\partial \eta}= & \frac{n}{\eta}+\sum_{i=1}^{n} \ln x_{i}+(\omega-1) \sum_{i=1}^{n} \frac{\gamma x_{i}^{\eta} \ln x_{i}}{1+\gamma x_{i}^{\eta}}
\end{aligned}
$$

$$
\begin{aligned}
& -\omega \gamma \sum_{i=1}^{n} x_{i}^{\eta} \ln x_{i}\left(1+\gamma x_{i}^{\eta}\right)^{\omega-1} \\
& +2 \omega \sum_{i=1}^{n} \frac{\gamma x_{i}^{\eta} \ln x_{i}\left(1+\gamma x_{i}^{\eta}\right)^{\omega-1} e^{1-\left(1+\gamma x_{i}^{\eta}\right)^{\omega}}}{1+e^{1-\left(1+\gamma x_{i}^{\eta}\right)^{\omega}}}=0 \\
\frac{\partial \ell}{\partial \gamma}= & \frac{n}{\gamma}+(\omega-1) \sum_{i=1}^{n} \frac{x_{i}^{\eta}}{1+\gamma x_{i}^{\eta}}-\omega \sum_{i=1}^{n}\left(1+\gamma x_{i}^{\eta}\right)^{\omega-1} x_{i}^{\eta} \\
& +2 \omega \sum_{i=1}^{n} \frac{x_{i}^{\eta}\left(1+\gamma x_{i}^{\eta}\right)^{\omega-1} e^{1-\left(1+\gamma x_{i}^{\eta}\right)^{\omega}}}{1+e^{1-\left(1+\gamma x_{i}^{\eta}\right)^{\omega}}}=0
\end{aligned}
$$

The above equations cannot be solved analytically, and statistical software can be used to solve them numerically via iterative methods and get the maximum likelihood estimate (MLE) of $\omega, \eta$, and $\gamma$.

4.1. Asymptotic Distribution. In order to have approximate confidence intervals (CIs) of the involved parameters, we require the estimated values of the elements of variancecovariance matrix $V$ of the MLEs. The variance-covariance matrix $V$ is estimated by the observed information matrix $\widehat{V}$, where

$$
\widehat{V}=-\left[\begin{array}{lll}
I_{11} & I_{12} & I_{13} \\
I_{21} & I_{22} & I_{23} \\
I_{31} & I_{32} & I_{33}
\end{array}\right],
$$


TABLE 2: Bias and MSE for the HLGW parameters.

\begin{tabular}{|c|c|c|c|c|c|c|c|c|c|}
\hline$\omega$ & $\eta$ & $\gamma$ & $n$ & $\operatorname{Bias}(\omega)$ & $\operatorname{MSE}(\omega)$ & $\operatorname{Bias}(\eta)$ & $\operatorname{MSE}(\eta)$ & $\operatorname{Bias}(\gamma)$ & $\operatorname{MSE}(\gamma)$ \\
\hline \multirow{7}{*}{1.5} & \multirow{7}{*}{5} & \multirow{7}{*}{0.5} & 20 & 0.2103 & 0.0392 & 1.1935 & 3.2415 & 0.3454 & 0.9104 \\
\hline & & & 40 & 0.1732 & 0.0314 & 1.1431 & 2.9381 & 0.2931 & 0.5830 \\
\hline & & & 60 & 0.1526 & 0.0283 & 1.1063 & 2.4897 & 0.2480 & 0.3148 \\
\hline & & & 80 & 0.1473 & 0.0231 & 0.9733 & 2.1738 & 0.1812 & 0.1908 \\
\hline & & & 100 & 0.1332 & 0.0207 & 0.8910 & 1.9318 & 0.1317 & 0.0813 \\
\hline & & & 250 & 0.1171 & 0.0113 & 0.5337 & 1.3877 & 0.0811 & 0.0031 \\
\hline & & & 500 & 0.1010 & 0.0061 & 0.2701 & 1.0814 & 0.0213 & 0.0008 \\
\hline \multirow{7}{*}{1.5} & \multirow{7}{*}{10} & \multirow{7}{*}{1} & 20 & 0.3118 & 0.0433 & 2.6754 & 15.9312 & 0.6125 & 10.2311 \\
\hline & & & 40 & 0.2918 & 0.0395 & 2.3487 & 12.3471 & 0.2451 & 7.6401 \\
\hline & & & 60 & 0.2554 & 0.0365 & 2.1174 & 9.2877 & 0.1615 & 4.3881 \\
\hline & & & 80 & 0.2375 & 0.0335 & 2.0968 & 6.2514 & 0.0941 & 1.9722 \\
\hline & & & 100 & 0.2114 & 0.0317 & 2.0532 & 4.9934 & 0.0532 & 1.5531 \\
\hline & & & 250 & 0.1783 & 0.0307 & 0.0065 & 1.9951 & 0.0113 & 0.4899 \\
\hline & & & 500 & 0.1265 & 0.0299 & 0.0013 & 0.2164 & 0.0095 & 0.0989 \\
\hline
\end{tabular}

where $I_{i j}, i, j=1,2,3$, are the second partial derivatives of (29) with respect to $\omega, \eta$, and $\gamma$. They are the entries of Fisher's information matrix analogous to $\omega, \eta$, and $\gamma$, respectively, which are given in Appendix. The diagonal of matrix in (31) gives the variances of the MLEs of $\omega, \eta$, and $\gamma$, respectively.

Approximation by a standard normal (SN) distribution of the distribution of $Z_{\widehat{\theta}_{k}}=\left(\widehat{\theta}_{k}-\theta_{k}\right) / \sqrt{\widehat{\operatorname{Var}}\left(\hat{\theta}_{k}\right)}$, where $\hat{\theta}=$ $(\widehat{\omega}, \widehat{\eta}, \widehat{\gamma})$, results in an approximate $100(1-\vartheta) \%$ confidence interval for $\theta_{k}$ as

$$
\widehat{\theta}_{k} \pm z_{\vartheta / 2} \sqrt{\widehat{\operatorname{Var}}\left(\widehat{\theta}_{k}\right)}, \quad j=1,2,3,
$$

where $z_{\vartheta / 2}$ is the upper $(\vartheta / 2) 100$ th percentile of SN distribution. We can use the likelihood ratio (LR) test to compare the fit of the HLGW distribution with its submodels for a given data set. For example, to test $\gamma=0$, the LR statistic is $\mathbf{w}=2[\ln (L(\widehat{\omega}, \widehat{\eta}, \widehat{\gamma}))-\ln (L(\widetilde{\omega}, \tilde{\eta}, 0))]$, where $\widehat{\omega}, \widehat{\eta}$, and $\widehat{\gamma}$ are the unrestricted estimates and $\widetilde{\omega}, \widetilde{\eta}$ are the restricted estimates. The LR test rejects the null hypothesis if $\mathbf{w}>\chi_{\epsilon}^{2}$, where $\chi_{\epsilon}^{2}$ denotes the upper $100 \in \%$ point of the $\chi^{2}$-distribution with 1 degree of freedom.

\section{Simulation Study}

The MLEs can be checked out by a simulation study. The following steps can be followed:

(1) By using (6), 5,000 samples of size $n$ are achieved. The variates of the HLGW distribution are developed using

$$
X=\left[\frac{1}{\gamma}\left\{\left(1-\ln \left(\frac{1-u}{1+u}\right)\right)^{1 / \omega}-1\right\}\right]^{1 / \eta},
$$

for $u \sim U(0,1)$.

(2) The MLEs are computed for the samples, say $\widehat{\Theta}_{j}=$ $\left(\widehat{\omega}_{j}, \widehat{\eta}_{j}, \widehat{\gamma}_{j}\right)$ for $j=1,2, \ldots, 5,000$.
(3) The mean square errors (MSEs) are calculated for every parameter.

The above steps were repeated for $n=20,40,60,80,100,250$, and 500 with $\omega=1.5, \eta=5, \gamma=0.5$ and $\omega=1.5, \eta=$ $10, \gamma=1$. Table 2 shows the bias and MSEs of $\omega, \eta$, and $\gamma$. It can be deduced through the table that MSEs for individual parameters fall to zero when sample size increases.

\section{Applications}

This section deals with the applications of the HLGW model to two lifetime data sets, that is, the data of 213 observed values of intervals between failures of air conditioning system of Boeing 720 jet airplanes and the data of waiting times (min) of 100 bank customers. Estimates of the parameters of HLGW distribution (standard errors in parentheses) and Cramervon Mises $W^{*}$, Anderson Darling $A^{*}$, and K-S statistics are presented for the data sets. In general, the smaller the values of $W^{*}, A^{*}$, and K-S statistics, the better the fit. We compare the proposed model with other models for the same data sets to check the potentiality and flexibility of new model.

6.1. Air Conditioning Systems Failure Data. The first application concerns 213 observed values of intervals between failures of air conditioning system of Boeing 720 jet airplanes firstly analysed by Proschan [16]. We have compared the performance of the HLGW distribution with its submodels as well as with some other well-known models given below.

The Weibull Poisson (WP) distribution by Lu and Shi [17] with the PDF:

$$
g(t ; \alpha, \beta, \lambda)=\frac{\alpha \beta \lambda t^{\alpha-1}}{1-e^{-\lambda}} e^{-\lambda-\beta t^{\alpha}+\lambda \exp \left(-\beta t^{\alpha}\right)}, \quad t>0
$$

for $\alpha, \beta$, and $\lambda>0$. 
TABLE 3: Estimates of models for the air conditioning systems failure data.

\begin{tabular}{|c|c|c|c|c|c|c|c|c|}
\hline \multirow{2}{*}{ Distributions } & \multicolumn{3}{|c|}{ Estimates } & \multirow{2}{*}{$-2 \ell(\widehat{\theta})$} & \multicolumn{4}{|c|}{ Statistics } \\
\hline & $\widehat{\omega}$ & $\widehat{\eta}$ & $\widehat{\gamma}$ & & $W^{*}$ & $A^{*}$ & K-S & $p$ value \\
\hline HLGW & $\begin{array}{c}0.3470 \\
(0.0885)\end{array}$ & $\begin{array}{c}1.3814 \\
(0.2462)\end{array}$ & $\begin{array}{c}0.0296 \\
(0.0127)\end{array}$ & 2349.674 & 0.0339 & 0.2587 & 0.0394 & 0.8957 \\
\hline HLW & & $\begin{array}{c}0.7804 \\
(0.0426)\end{array}$ & $\begin{array}{c}0.0442 \\
(0.0098)\end{array}$ & 2360.630 & 0.2099 & 1.2851 & 0.0577 & 0.4770 \\
\hline HLE & & & $\begin{array}{c}0.0144 \\
(0.0009)\end{array}$ & 2383.325 & 0.3446 & 2.0606 & 0.1407 & 0.0004 \\
\hline HLNH & $\begin{array}{c}0.5596 \\
(0.0515)\end{array}$ & & $\begin{array}{c}0.0482 \\
(0.0108)\end{array}$ & 2352.930 & 0.0850 & 0.5534 & 0.0445 & 0.7926 \\
\hline Weibull & $\begin{array}{c}0.0158 \\
(0.0038)\end{array}$ & $\begin{array}{c}0.9226 \\
(0.0459)\end{array}$ & & 2355.171 & 0.1373 & 0.8552 & 0.0514 & 0.6270 \\
\hline CWG & $\begin{array}{c}0.2890 \\
(0.0238)\end{array}$ & $\begin{array}{c}0.0079 \\
(0.0032)\end{array}$ & $\begin{array}{c}4.1050 \\
(2.8847)\end{array}$ & 2362.329 & 0.1519 & 1.0253 & 0.0502 & 0.6563 \\
\hline WP & $\begin{array}{c}0.4015 \\
(0.0584)\end{array}$ & $\begin{array}{c}0.4967 \\
(0.1838)\end{array}$ & $\begin{array}{l}-8.0978 \\
(2.5766)\end{array}$ & 2351.850 & 0.0440 & 0.3305 & 0.0412 & 0.8624 \\
\hline
\end{tabular}

The complementary Weibull geometric (CWG) distribution by Tojeiro et al. [18] with the PDF:

$$
g(t)=\frac{\gamma \lambda^{\gamma} \theta t^{\gamma-1} e^{-(\lambda t)^{\gamma}}}{\left[\theta+(1-\theta) e^{-(\lambda t)^{\gamma}}\right]^{2}},
$$

for $t, \lambda, \gamma$, and $\theta>0$.

The Weibull (W) distribution with PDF:

$$
g(y ; \beta, \gamma)=\beta \gamma y^{\gamma-1} \exp \left(-\beta y^{\gamma}\right)
$$

for $y>0, \beta>0$, and $\gamma>0$.

The estimated values of the parameters with standard errors (SE) are found using the method of maximum likelihood estimation. Table 3 gives the estimated values of the parameters along with their standard errors and the test statistics have the smallest values of $W^{*}, A^{*}$, and K-S statistic for the data set under HLGW distribution as compared to the other models. Based on these values, it is concluded that HLGW distribution is the best model as compared to the other models to fit this data set. This conclusion can also be made by the CDF plots for empirical and fitted HLGW distributions using data of 213 values of intervals between failures of air conditioning system in Figure 3(a) for the data. In Figure 3(b), the TTT plot is shown for the data set. The failure rate shape for this data set is decreasing, as its TTT plot is convex.

The LR test statistics of hypotheses $H_{0}$ : HLW versus $H_{a}$ : HLGW, $H_{0}$ : HLE versus $H_{a}$ : HLGW, and $H_{0}$ : HLNH versus $H_{a}$ : HLGW are 10.956 ( $p$ value $\left.=0.00093\right), 33.651$ ( $p$ value $=0.00001)$, and 3.256 ( $p$ value $=0.071163)$, respectively. We conclude that there is a significant difference between HLW and HLGW distributions, HLE and HLGW, and also between HLNH and HLGW distributions at the $10 \%$ level.

6.2. Waiting Time Data. The data encountered in the second application involves data of waiting times (min) of 100 bank customers used by Bidram and Nadarajah [4]. We have compared the performance of the HLGW distribution with its submodels as well as with some other well-known distributions, such as the Weibull and the complementary Weibull geometric distribution. We also use the LR test to compare the HLGW distribution and its submodels.

The estimated values of the parameters, the standard errors, and the goodness-of-fit test statistics $W^{*}, A^{*}$, and K-S statistic for the data of waiting time are given in Table 4 . These tables illustrate that HLGW model shows a good fit for this data set as compared to the other distributions. The CDF plot for empirical and HLGW distributions using the data set of waiting time in Figure 4(a) also confirms the fitness of HLGW distribution. Figure 4(b) expresses the TTT plot for this data set. Since the TTT plot of the data set is concave, the data set has increasing hazard rate shape. The LR test statistics of the hypotheses $H_{0}$ : HLW versus $H_{a}$ : HLGW, $H_{0}$ : HLE versus $H_{a}$ : HLGW, and $H_{0}$ : HLNH versus $H_{a}$ : HLGW are 5.501 ( $p$ value $=0.019006), 11.85(p$ value $=0.000577)$, and $9.271(p$ value $=$ $0.002328)$. The HLGW distribution is significantly better than HLW, HLE, and HLNH distributions. There is no difference between HLGW and Weibull distribution based on the LR test; however the goodness-of-fit statistics $W^{*}, A^{*}$, and K-S statistic clearly show that HLGW distribution is better than Weibull distribution for the data.

\section{Conclusion}

We have proposed a three-parameter lifetime generalized distribution, referred to as the half-logistic generalized Weibull (HLGW) distribution. The HLGW distribution has three other distributions like the half-logistic exponential, the halflogistic Weibull, and the half-logistic Nadarajah-Haghighi distributions, as its submodels. The new model exhibits a variety of shapes of the failure rate function, that is, increasing, increasing and then constant, decreasing, bathtub, unimodal, and decreasing-increasing-decreasing (DID) shapes. Various statistical properties of the HLGW distribution are derived and studied in detail. We estimated the parameters involved 


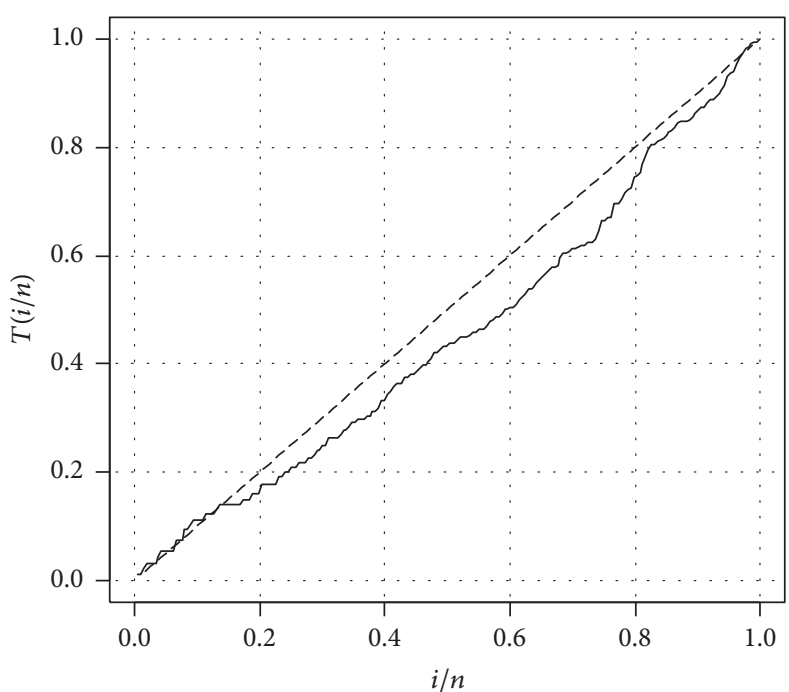

(a) TTT plot

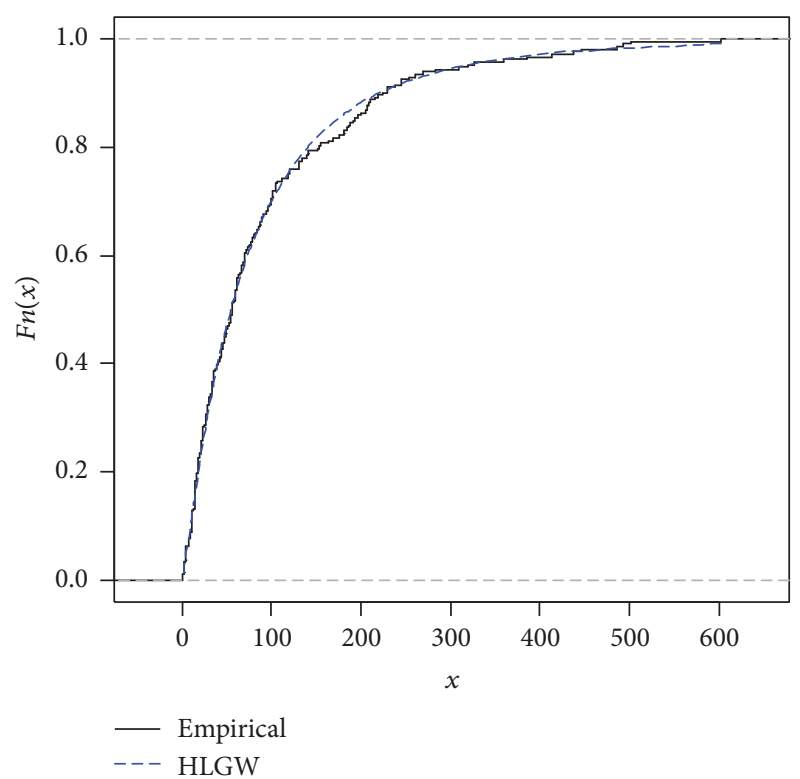

(b) CDF Plot

FIGURE 3: TTT plot and CDF plot for the air conditioning systems failure data.

TABLE 4: Estimates of models for the waiting time data.

\begin{tabular}{|c|c|c|c|c|c|c|c|c|}
\hline \multirow{2}{*}{ Distributions } & \multicolumn{3}{|c|}{ Estimates } & \multirow{2}{*}{$-2 \ell(\widehat{\theta})$} & \multicolumn{4}{|c|}{ Statistics } \\
\hline & $\widehat{\omega}$ & $\widehat{\eta}$ & $\widehat{\gamma}$ & & $W^{*}$ & $A^{*}$ & K-S & $p$ value \\
\hline HLGW & $\begin{array}{c}0.3610 \\
(0.1268)\end{array}$ & $\begin{array}{c}2.1425 \\
(0.5257)\end{array}$ & $\begin{array}{c}0.0773 \\
(0.0340)\end{array}$ & 634.226 & 0.0176 & 0.1277 & 0.0368 & 0.9992 \\
\hline HLW & & $\begin{array}{c}1.2336 \\
(0.0978)\end{array}$ & $\begin{array}{c}0.0772 \\
(0.0216)\end{array}$ & 639.730 & 0.0934 & 0.5740 & 0.0627 & 0.8267 \\
\hline HLE & & & $\begin{array}{c}0.1446 \\
(0.0119)\end{array}$ & 646.076 & 0.0583 & 0.3624 & 0.1213 & 0.1053 \\
\hline HLNH & $\begin{array}{c}1.4044 \\
(0.3411)\end{array}$ & & $\begin{array}{c}0.0846 \\
(0.0316)\end{array}$ & 643.497 & 0.1032 & 0.6355 & 0.0877 & 0.4257 \\
\hline Weibull & $\begin{array}{c}0.0305 \\
(0.0095)\end{array}$ & $\begin{array}{c}1.4582 \\
(0.1089)\end{array}$ & & 637.461 & 0.0629 & 0.3961 & 0.0577 & 0.8938 \\
\hline CWG & $\begin{array}{c}0.5066 \\
(0.0779) \\
\end{array}$ & $\begin{array}{c}0.0126 \\
(0.0097) \\
\end{array}$ & $\begin{array}{c}2.2254 \\
(1.7142) \\
\end{array}$ & 645.742 & 0.0745 & 0.4653 & 0.0485 & 0.9726 \\
\hline
\end{tabular}

in the model by using the method of MLEs. Two real data sets are used to illustrate the flexibility, potentiality, and usefulness of HLGW distribution. It is concluded that HLGW model delivers better fitting than the other lifetime models and we hope that HLGW distribution may attract wider range of practical applications and this research may serve as a reference and benefit future research in the subject field of study.

\section{Appendix}

\section{Fisher's Information Matrix}

The elements of Fisher's information matrix analogous to $\omega$, $\eta$, and $\gamma$ :

$$
\begin{aligned}
& \frac{\partial^{2} \ell}{\partial \omega^{2}}=-\frac{n}{\omega^{2}}-\sum_{i=1}^{n} A_{i}^{\omega}\left(\ln A_{i}\right)^{2} \\
& +2 \sum_{i=1}^{n} \frac{\left(\ln A_{i}\right)^{2} A_{i}^{\omega} e^{1-A_{i}^{\omega}}}{\left(1+e^{1-A_{i}^{\omega}}\right)^{2}}\left(1-A_{i}^{\omega}+e^{1-A_{i}^{\omega}}\right) \\
& \frac{\partial^{2} \ell}{\partial \omega \partial \eta}=\frac{\partial^{2}}{\partial \eta \partial \omega}=\sum_{i=1}^{n} \frac{\gamma x_{i}^{\eta} \ln x_{i}}{A_{i}}\left[1-A_{i}^{\omega}\left(1+\omega \ln A_{i}\right)\right.
\end{aligned}
$$

$$
\begin{aligned}
& +\frac{2 A_{i}^{\omega} e^{1-A_{i}^{\omega}}}{1+e^{1-A_{i}^{\omega}}} \\
& \left.+\frac{2 \omega A_{i}^{\omega} \ln A_{i} e^{1-A_{i}^{\omega}}}{\left(1+e^{1-A_{i}^{\omega}}\right)^{2}}\left(1-A_{i}^{\omega}+e^{1-A_{i}^{\omega}}\right)\right]
\end{aligned}
$$




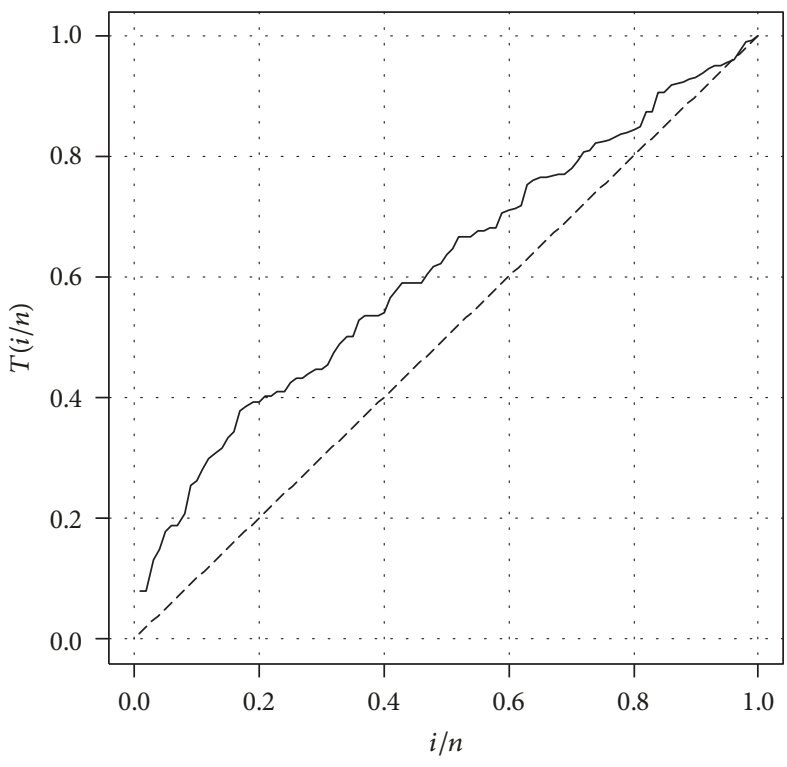

(a) TTT plot

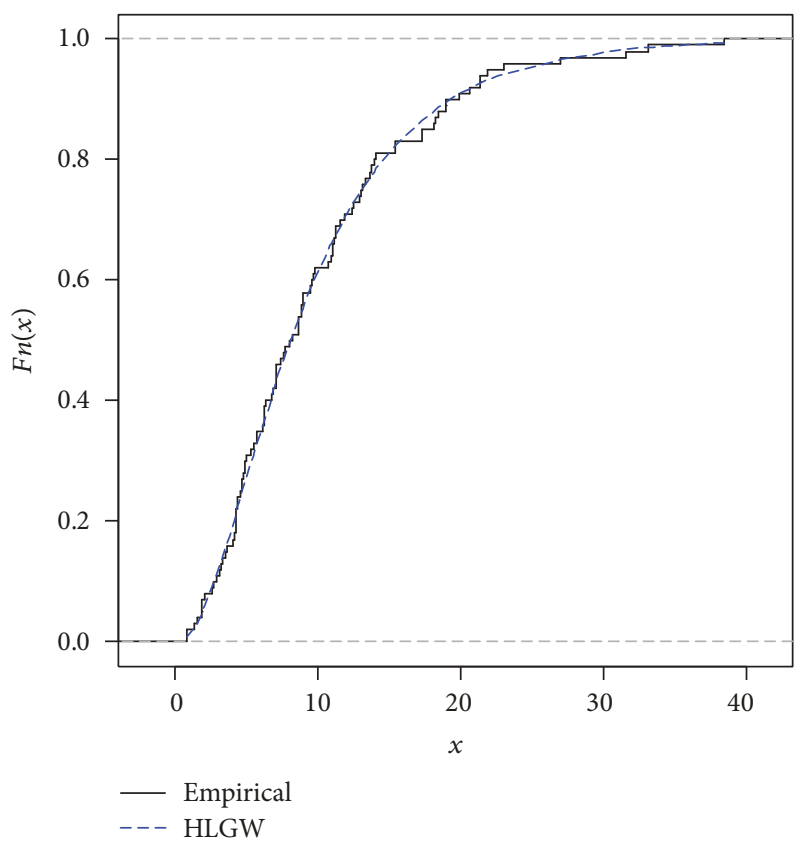

(b) CDF Plot

FIGURE 4: TTT plot and CDF plot for the waiting time data.

$$
\begin{aligned}
& \frac{\partial^{2} \ell}{\partial \omega \partial \gamma}=\frac{\partial^{2} \ell}{\partial \gamma \partial \omega}=\sum_{i=1}^{n} \frac{x_{i}^{\eta}}{A_{i}}\left[1-A_{i}^{\omega}\left(1+\omega \ln A_{i}\right)\right. \\
& +\frac{2 A_{i}^{\omega} e^{1-A_{i}^{\omega}}}{1+e^{1-A_{i}^{\omega}}} \\
& \left.+\frac{2 \omega A_{i}^{\omega} \ln A_{i} e^{1-A_{i}^{\omega}}}{\left(1+e^{1-A_{i}^{\omega}}\right)^{2}}\left(1-A_{i}^{\omega}+e^{1-A_{i}^{\omega}}\right)\right] \\
& \frac{\partial^{2} \ell}{\partial \eta^{2}}=-\frac{n}{\eta^{2}}+\sum_{i=1}^{n} \frac{\gamma x_{i}^{\eta}\left(\ln x_{i}\right)^{2}}{A_{i}}\left[-\omega A_{i}^{\omega}+(\omega-1)\right. \\
& \cdot\left(1-\frac{\gamma x_{i}^{\eta}}{A_{i}}-\frac{\omega A_{i}^{\omega} \gamma x_{i}^{\eta}}{A_{i}}+\frac{2 \omega A_{i}^{\omega} \gamma x_{i}^{\eta} e^{1-A_{i}^{\omega}}}{A_{i}\left(1+e^{1-A_{i}^{\omega}}\right)}\right) \\
& \left.+\frac{2 \omega A_{i}^{\omega} e^{1-A_{i}^{\omega}}}{1+e^{1-A_{i}^{\omega}}}\left(1-\frac{\omega A_{i}^{\omega} \gamma x_{i}^{\eta}}{A_{i}\left(1+e^{1-A_{i}^{\omega}}\right)}\right)\right] \\
& \frac{\partial^{2} \ell}{\partial \eta \partial \gamma}=\frac{\partial^{2} \ell}{\partial \gamma \partial \eta}=\sum_{i=1}^{n} \frac{x_{i}^{\eta} \ln x_{i}}{A_{i}}\left[-\omega A_{i}^{\omega}+(\omega-1)\right. \\
& \cdot\left(1-\frac{\gamma x_{i}^{\eta}}{A_{i}}-\frac{\omega A_{i}^{\omega} \gamma x_{i}^{\eta}}{A_{i}}+\frac{2 \omega A_{i}^{\omega} \gamma x_{i}^{\eta} e^{1-A_{i}^{\omega}}}{A_{i}\left(1+e^{1-A_{i}^{\omega}}\right)}\right) \\
& \left.+\frac{2 \omega A_{i}^{\omega} e^{1-A_{i}^{\omega}}}{1+e^{1-A_{i}^{\omega}}}\left(1-\frac{\omega A_{i}^{\omega} \gamma x_{i}^{\eta}}{A_{i}\left(1+e^{1-A_{i}^{\omega}}\right)}\right)\right] \\
& \frac{\partial^{2} \ell}{\partial \gamma^{2}}=-\frac{n}{\gamma^{2}}-\sum_{i=1}^{n} \frac{x_{i}^{2 \eta}}{A_{i}^{2}}[(\omega-1)
\end{aligned}
$$$$
\left.\cdot\left(1+\omega A_{i}^{\omega}-\frac{2 \omega A_{i}^{\omega} e^{1-A_{i}^{\omega}}}{1+e^{1-A_{i}^{\omega}}}\right)+\frac{2 \omega^{2} A_{i}^{2 \omega} e^{1-A_{i}^{\omega}}}{\left(1+e^{1-A_{i}^{\omega}}\right)^{2}}\right] .
$$

\section{Conflicts of Interest}

The authors declare that they have no conflicts of interest.

\section{References}

[1] C. D. Lai, "Constructions and applications of lifetime distributions," Applied Stochastic Models in Business and Industry, vol. 29, no. 2, pp. 127-140, 2013.

[2] A. W. Marshall and I. Olkin, "A new method for adding a parameter to a family of distributions with application to the exponential and Weibull families," Biometrika, vol. 84, no. 3, pp. 641-652, 1997.

[3] B. Al-Zahrani and H. Sagor, "The Poisson-Lomax distribution," Revista Colombiana de Estadistica, vol. 37, no. 1, pp. 225-245, 2014.

[4] H. Bidram and S. Nadarajah, "A new lifetime model with decreasing, increasing, bathtub-shaped, and upside-down bathtub-shaped hazard rate function," Statistics: A Journal of Theoretical and Applied Statistics, 2015.

[5] C. Kus, "A new lifetime distribution," Computational Statistics \& Data Analysis, vol. 51, no. 9, pp. 4497-4509, 2007.

[6] M. R. Gurvich, A. T. DiBenedetto, and S. V. Ranade, "A new statistical distribution for characterizing the random strength of brittle materials," Journal of Materials Science, vol. 32, no. 10, pp. 2559-2564, 1997.

[7] S. Nadarajah and S. Kotz, "On some recent modifications of Weibull distribution," IEEE Transactions on Reliability, vol. 54, no. 4, pp. 561-562, 2005. 
[8] C. D. Lai, M. Xie, and D. N. P. Murthy, "Reply to "On some recent modifications of Weibull distribution"," IEEE Transactions on Reliability, vol. 54, no. 4, p. 563, 2005.

[9] C. D. Lai, M. Xie, and D. N. P. Murthy, "A modified Weibull distribution," IEEE Transactions on Reliability, vol. 52, no. 1, pp. 33-37, 2003.

[10] M. Xie, Y. Tang, and T. N. Goh, "A modified Weibull extension with bathtub-shaped failure rate function," Reliability Engineering \& System Safety, vol. 76, no. 3, pp. 279-285, 2002.

[11] T. Dimitrakopoulou, K. Adamidis, and S. Loukas, "A lifetime distribution with an upside-down bathtub-shaped hazard function," IEEE Transactions on Reliability, vol. 56, no. 2, pp. 308-311, 2007.

[12] G. M. Cordeiro, M. Alizadeh, and P. . Marinho, "The type I half-logistic family of distributions," Journal of Statistical Computation and Simulation, vol. 86, no. 4, pp. 707-728, 2016.

[13] S. D. Krishnarani, "On a power transformation of half-logistic distribution," Journal of Probability and Statistics, vol. 2016, Article ID 2084236, 2016.

[14] J. Kenney and E. S. Keeping, Mathematics of Statistics, Van Nostrand, Princeton, NJ, USA, 1962, Van Nostrand., Mathematics of Statistics.

[15] J. J. Moors, "A Quantile Alternative for Kurtosis," The American Statistician, vol. 37, no. 1, p. 25, 1988.

[16] F. Proschan, "Theoretical Explanation of Observed Decreasing Failure Rate," Technometrics, vol. 5, no. 3, pp. 375-383, 1963.

[17] W. Lu and D. Shi, "A new compounding life distribution: the Weibull-Poisson distribution," Journal of Applied Statistics, vol. 39, no. 1, pp. 21-38, 2012.

[18] C. Tojeiro, F. Louzada, M. Roman, and P. Borges, "The complementary Weibull geometric distribution," Journal of Statistical Computation and Simulation, vol. 84, no. 6, pp. 1345-1362, 2014. 


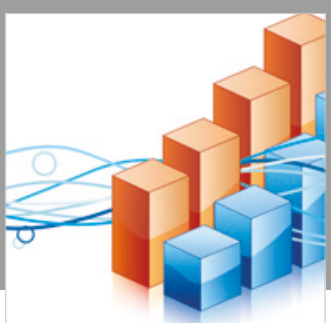

Advances in

Operations Research

\section{-n-m}
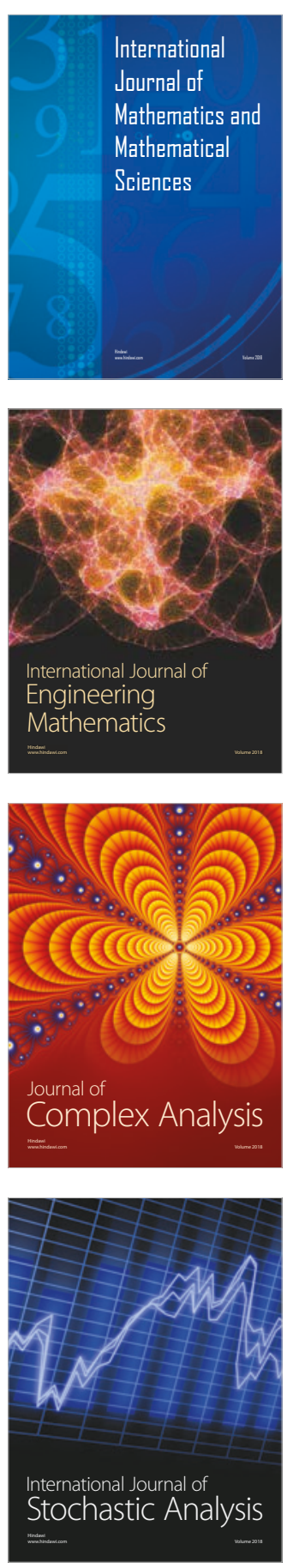
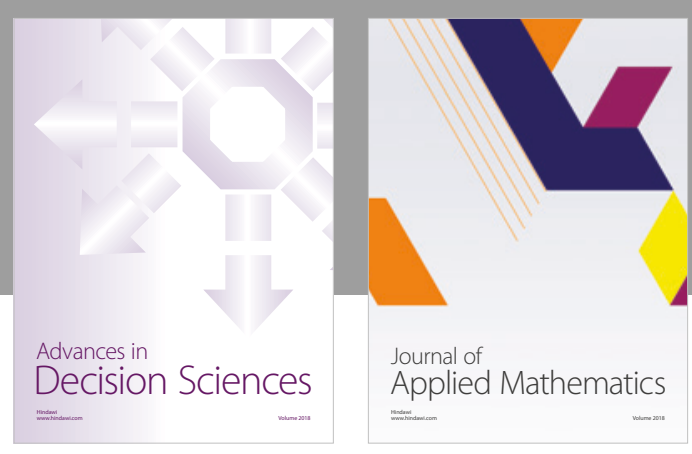

Journal of

Applied Mathematics
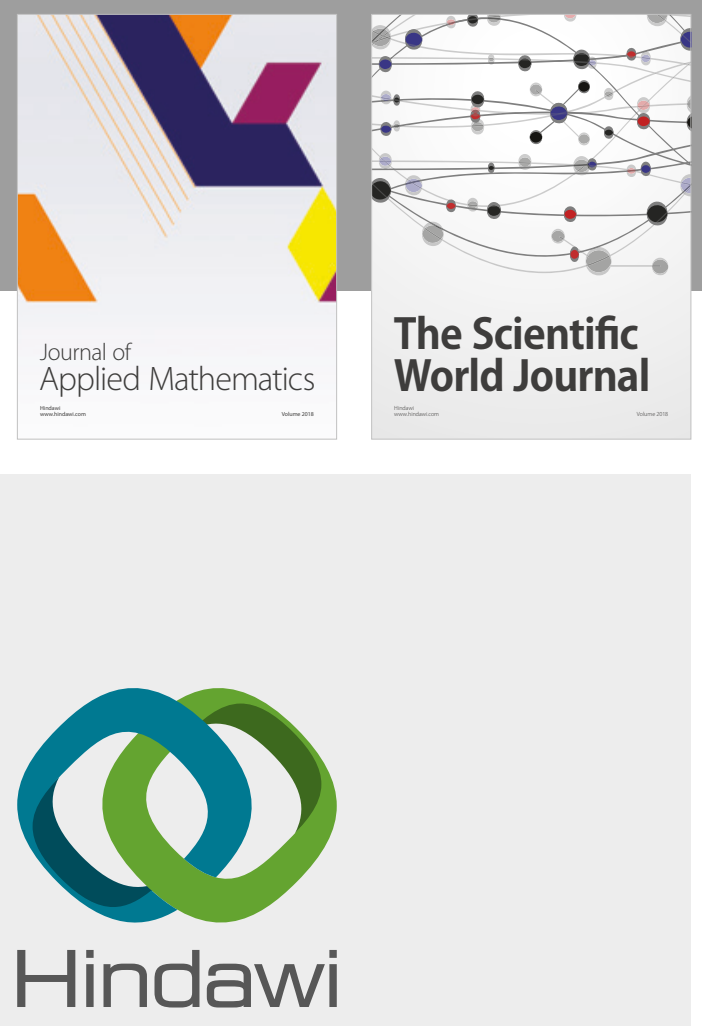

Submit your manuscripts at

www.hindawi.com

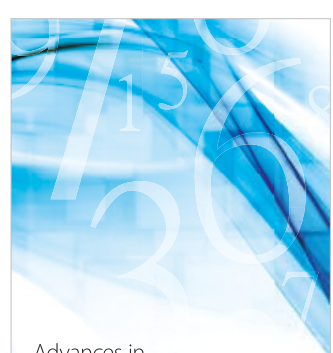

Advances in
Numerical Analysis
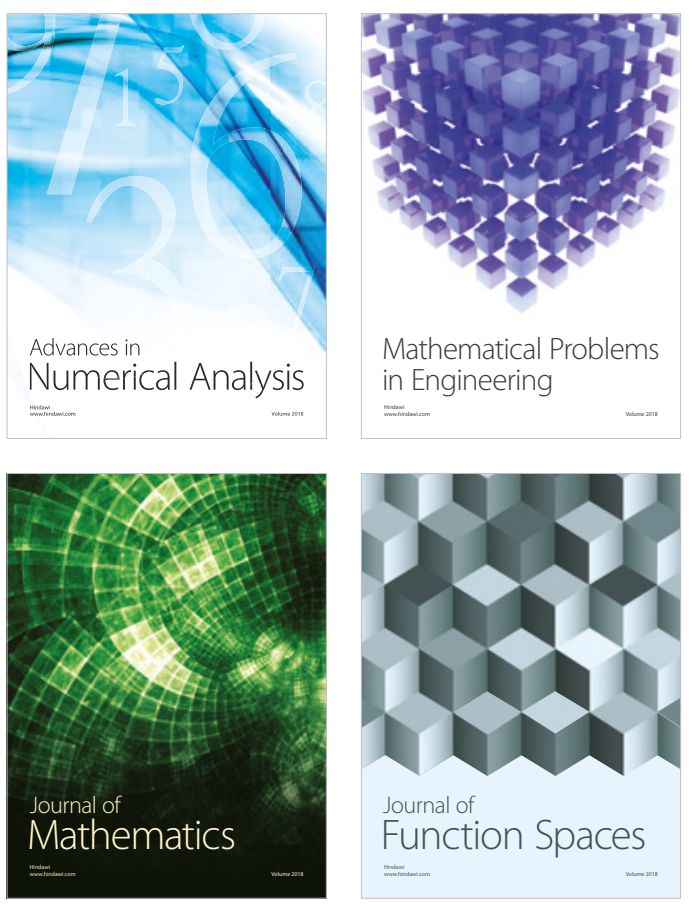

Mathematical Problems in Engineering

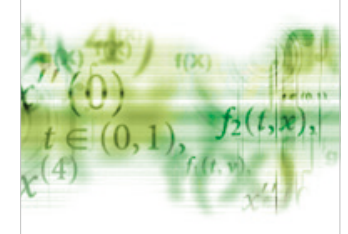

International Journal of

Differential Equations

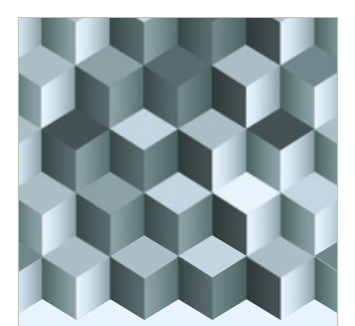

Journal of

Function Spaces

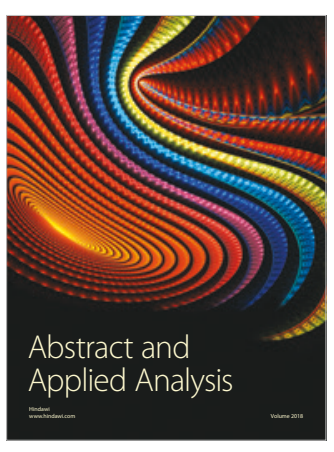

The Scientific

World Journal

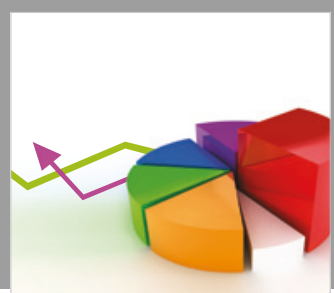

Journal of

Probability and Statistics
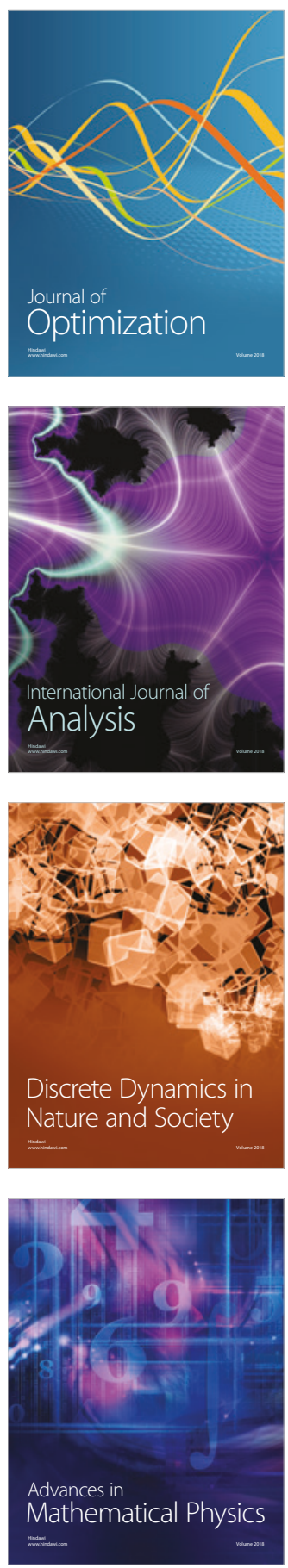